\title{
Identifying long non-coding RNAs and characterizing their functional roles in swine mammary gland from colostrogenesis to lactogenesis
}

\author{
Lijun Shi ${ }^{1}$, Longchao Zhang ${ }^{1}$, Ligang Wang ${ }^{1}$, Xin Liu $^{1}$, Hongmei Gao ${ }^{1}$, Xinhua Hou ${ }^{1}$, \\ Fuping Zhao ${ }^{1}$, Hua Yan ${ }^{1}$, Wentao Cai ${ }^{1 \star *}$, and Lixian Wang ${ }^{1, *}$
}

\section{* Corresponding Authors: \\ Wentao Cai \\ Tel: +86-010-62816065, \\ E-mail:wtaocai@gmail.com \\ Lixian Wang \\ Tel: +86-010-62818771, \\ E-mail: iaswlx@263.net}

'Institute of Animal Science, Chinese Academy of Agricultural Sciences, Beijing 100193, China

ORCID

Lijun Shi

https://orcid org/0000-0003-4156-5123 Longchao Zhang

https://orcid.org/0000-0002-4520-3165 Ligang Wang

https://orcid.org/0000-0002-4376-8373

Xin Liu

https://orcid.org/0000-0001-7146-779X

Hongmei Gao

https://orcid.org/0000-0001-9237-439X Xinhua Hou

https://orcid.org/0000-0001-9485-7766

Fuping Zhao

https://orcid.org/0000-0001-9582-5993

Hua Yan

https://orcid.org/0000-0001-5480-8513

Wentao Cai

https://orcid.org/0000-0002-5639-1755

Lixian Wang

https://orcid.org/0000-0001-8943-4697

Submitted Jul 6, 2021; Revised Aug 13, 2021; Accepted Sept 11, 2021

\begin{abstract}
Objective: This study was conducted to identify the functional long non-coding RNAs (lncRNAs) for swine lactation by RNA-seq data of mammary gland.

Methods: According to the RNA-seq data of swine mammary gland, we screened lncRNAs, performed differential expression analysis, and confirmed the functional lncRNAs for swine lactation by validation of genome wide association study (GWAS) signals, functional annotation and weighted gene co-expression network analysis (WGCNA).

Results: We totally identified 286 differentially expressed (DE) lncRNAs in mammary gland at different stages from 14 days prior to (-) parturition to day 1 after $(+)$ parturition, and the expressions of most of lncRNAs were strongly changed from day -2 to day +1 . Further, the GWAS signals of sow milk ability trait were significantly enriched in DE lncRNAs. Functional annotation revealed that these DE IncRNAs were mainly involved in mammary gland and lactation developing, milk composition metabolism and colostrum function. By performing weighted WGCNA, we identified 7 out of $12 \operatorname{lncRNA-mRNA}$ modules that were highly associated with the mammary gland at day -14 , day -2 , and day +1 , in which, 35 lncRNAs and $319 \mathrm{mRNAs}$ were involved.

Conclusion: This study suggested that $18 \operatorname{lncRNAs}$ and their 20 target genes were promising candidates for swine parturition and colostrum occurrence processes. Our research provided new insights into lncRNA profiles and their regulating mechanisms from colostrogenesis to lactogenesis in swine.
\end{abstract}

Keywords: Colostrum; LncRNA; Mammary Gland; Pig; Weighted Gene Co-expression Network Analysis (WGCNA)

\section{INTRODUCTION}

Although litter size of sow has made a significant improvement during last two decades, the problem of growth performance and mortality in piglets has become prominent. During gestation and lactation, the growth performance and health status of piglets have been critical factors impacting reproductive performance of modern sows [1]. Sow milk performance is a major limiting factor and contributes to suboptimal growth and survival of piglets [2]. The quality and quantity of milk in sows are highly variable, for example, the colostrum yield is reported to range from $<1 \mathrm{~kg}$ to $8.5 \mathrm{~kg}[3,4]$. To enhance the sow lactation ability, it is necessary to understand the mammary gland development, and screen their regulatory factors [5]. In 2016, Balzani et al [6] reported the heritability of udder morphology in crossbreed sows (Large WhitexMeishan), namely, $h^{2}: 0.1$ to 0.56 . Generally, from colostrogenesis to lactogenesis, mammary glands might undergo significant functional differentiation for swine parturition and colostrum occurrence processes. In 2018, 
Palombo et al [7] used the RNA sequencing (RNA-seq) of mammary gland to detect the candidate genes impacting swine parturition and lactation in crossbred sows (Danish Landrace $\times$ Yorkshire).

Long non-coding RNA (lncRNA) is a recently identified class of non-protein coding transcripts in eukaryotes with a minimum length of $200 \mathrm{nt}$ [8], and it plays an important regulatory role in biological processes [9]. Many $\operatorname{lncRNAs}$ have been reported along with the depth and quality of RNAseq [10]. In swine, many potential regulatory lncRNAs have been identified from various tissues, such as intramuscular adipose [11,12], longissimus dorsi muscle [13], preadipocytes [14], and porcine endometrium [15]. In 2018, Liang et al [9] built a systematic Sus scrofa lncRNA database named lncRNAnet that contained 53,468 S. scrofa lncRNAs with their sequence characteristics, genomic locations, conservation, overlapping single nucleotide polymorphism (SNP) and quantitative trait loci, and transcript abundance across nine tissues (fat, heart, kidney, liver, lung, muscle, ovarium, spleen, and testis). However, the identification of lncRNAs regulating milk traits from sow mammary gland is still lacking.

In present study, we identified lncRNAs of sow mammary gland at different stages from 14 days prior to parturition to day 1 after parturition according to the published RNA-seq data, and detected the differentially expressed (DE) lncRNAs. Further, validation of genome wide association study (GWAS) signals, functional annotation and weighted gene co-expression network analysis (WGCNA) were conducted to predict the regulatory functions of DE lncRNAs. Our results will pave the way for a better understanding of IncRNA functions in swine parturition and colostrum occurrence processes.

\section{MATERIALS AND METHODS}

\section{Ethic statement}

The data (BioProject ID: GSE101983) was in the Gene Expression Omnibus database.

\section{RNA-seq dataset}

The RNA-seq data sources (GSE101983) were involved in 15 mammary gland tissues, which were collected from 3 crossbred sows (Danish LandracexYorkshire) at days 14, 10, 6, and 2 before (-) parturition and day 1 after $(+)$ parturition (Supplementary Table S1)[7].

\section{Identification of IncRNAs}

Clean reads were obtained by removing adapter molecules, reads including poly-N, and low-quality reads using Trimmomatic (0.39) with defaults parameters [16]. All follow-up analyses were performed with the high-quality reads. Through STAR software (2.7.3a), we aligned clean reads to the pig ref- erence genome (Sscrofa11.1).

We used StringTie (1.3.5) and Scripture (beta2) to assemble the novel transcripts. Transcripts occurred in at least two samples or supported by two assembly software were obtained $[17,18]$. Further, the putative novel lncRNAs were detected via a customized multi-step pipeline: i) The transcripts were removed, which were likely to be assembly artifacts or polymerase chain reaction run-on fragments based on class code annotated by gffcompare (v0.11.6). For the different class, we retained those only annotated by ' $u$ ' and ' $i$, which indicated novel intergenic and intronic transcripts, respectively. Transcripts with class code " $=$ " were considered as known genes. ii) Transcripts with length $\geq 200 \mathrm{nt}$ and exon $\geq 2$ were retained to avoid incomplete assemble and too many splicing events. iii) Transcripts with low expression were removed using fragments per kilobase of exon model per million mapped fragments (FPKM) $\geq 0.3$ as cut off. The expression levels of transcripts were calculated in fragments per kilo-base of exon per $10^{6}$ mapped fragments (FPKM) by StringTie -e -B. iv) Maximum open reading frame lengths of less than 120 amino acids (360 nt) were obtained. v) Transcripts with predicted protein-coding potential were removed, that the protein-coding potential criteria were CPC score $>0$, PLEK score $>0$, and CNCI score $>0$. Finally, the candidate sets of IncRNAs were made up by those without coding potentials. The process for identifying $\operatorname{lncRNAs}$ is shown in Figure 1A.

\section{Differential expression analysis of lncRNAs}

To identify the longitudinal transcriptional lncRNAs response close to parturition, and to highlight the metabolic processes underlying mammary changes associated with the colostrogenesis and lactogenesis in the last stages of gestation leading up to parturition, we used the time point -14 day as baseline to detect DE lncRNAs and coding genes with EdegR R package. RNA-Seq read counts were modeled by a generalized linear model that considered the experimental design with two factors (individuals and stages of lactation). The formula in EdegR is shown as follow: Design $=\sim$ Individuals + Stages . LncRNAs or genes with adjusted $p$-value $<0.05$ were assigned as the differences. Further, we performed expression patterns of DE lncRNAs across five stages by k-mean method [19].

Validation of genome wide association study signals We collected milk ability phenotypes of 985 sows from Shanxi and Liaoning Province of China in 2019 through 2020. Their genotypes were measuring using GenSeek Genomic Profiler Porcine 50K (50,697 SNPs), Illumina, San Diego, CA, USA), which contained 50,697 SNPs. The milk ability was calculated by the following formula: litter weight at weaning - litter weight at born - litter weight at the time of fostering in + litter weight at death + litter weight at the time of fostering out. We 

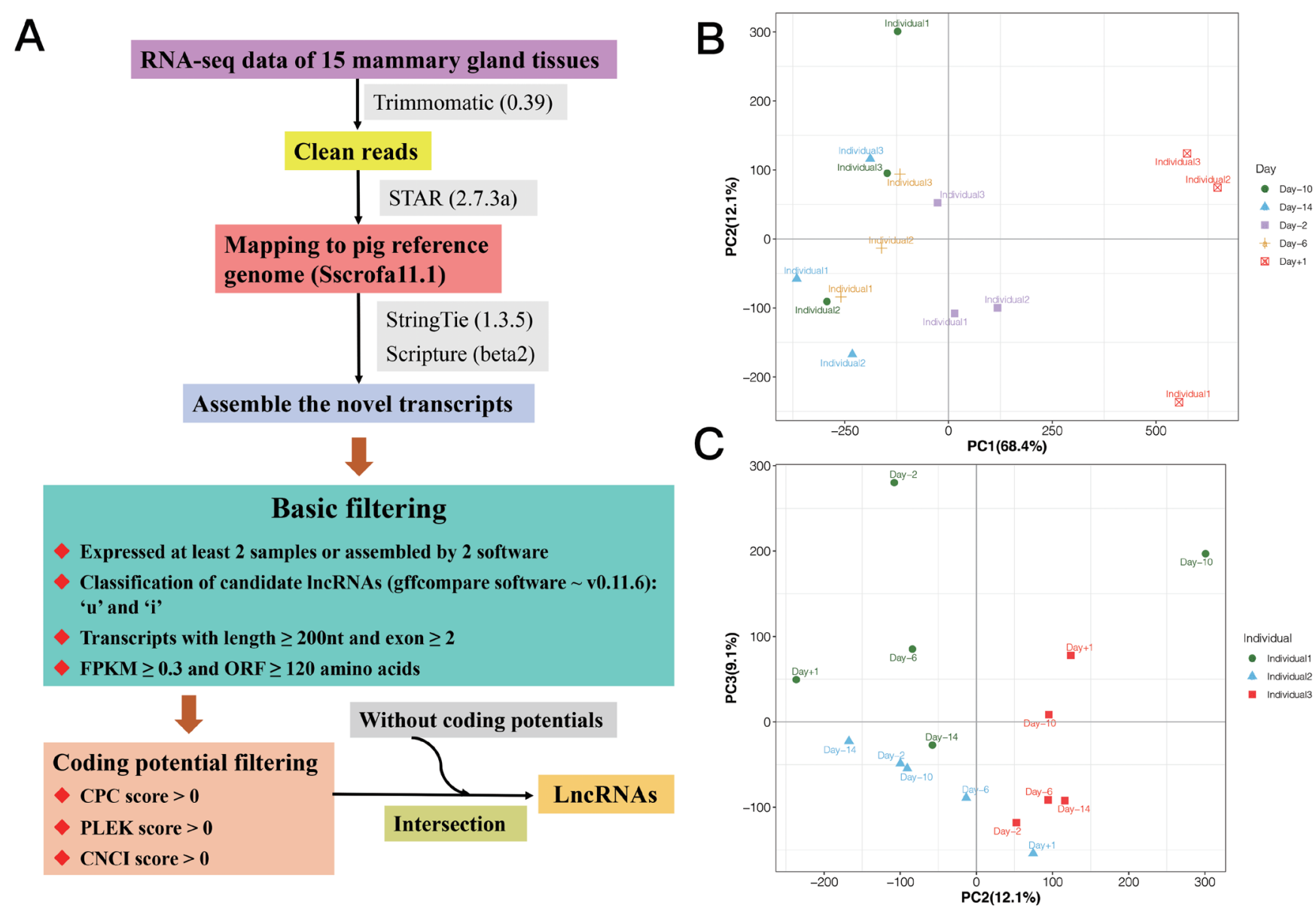

Figure 1. (A) The process for identifying IncRNAs. Principal component analysis (PCA) analysis based on the expression of IncRNAs: (B) PC1 and PC2 could separate the samples by phenotype of stages of lactation; and (C) PC2 and PC3 could separate the samples by individuals.

estimated the breeding value of milk produce ability with ASReml package, in which, fixed effects of herd by farm and production batch (9 levels), parity (5 levels: $1,2,3,4$, and 5-8), and days of lactation ( 3 levels: $\leq 18,19$ to 21 , and $>21$ ) were considered. We conducted the quality control for genotyping data by removing the SNPs with minor allele frequencies $<0.01$, and a deviation from Hardy-Weinburg equilibrium $p$ values $<0.001$. Finally, 36,871 SNPs and 985 phenotypic breeding values were used for GWAS by the Fixed and random effect model Circulating Probability Unification (FarmCPU). The genotype data used for GWAS and effects of SNPs were submitted to public repositories, and DOI was $10.6084 / \mathrm{m}$. figshare.16455963 (https://figshare.com/s/2112a49f046dfda $5 \mathrm{~d} 41 \mathrm{f})$.

For investigating the association of DE lncRNAs with GWAS signals, we performed enrichment analysis of SNPs from GWAS for sow milk ability trait (GitHub: https://github. com/WentaoCai/GWAS_enrichment) [20]. We separately conducted GWAS signals enrichment analysis based on 1,084 identified $\operatorname{lncRNAs}$ and $286 \mathrm{DE} \operatorname{lncRNAs}$ regions using a sumbased method. The formula was the following:

$$
T_{\text {sum }}=\sum_{i=1}^{m_{g}} \beta^{2}
$$

Where, $\boldsymbol{T}_{\text {sum }}$ was the summary statistics for a tested $\operatorname{lncRNA}$ group, $\beta$ was the marker effect of milk ability estimated by the GWAS statistics, and $\boldsymbol{m}_{g}$ was the number of SNPs located in the tested lncRNAs or $10 \mathrm{~Kb}$ up-/down-stream of the tested lncRNAs, in which, there were 1,571 and 485 SNPs for 1,084 and $286 \operatorname{lncRNAs}$, respectively. We repeated the permutation for 10,000 times in each studied genomic feature and calculated an empirical p-value according to one-tailed test of the proportion of randomly sampled summary statistics larger than that observed.

\section{Target gene prediction of lncRNAs and functional analysis}

LncRNAs can cis-regulate neighboring target genes and transregulate distant target genes [21,22]. To predict cis-regulated target genes of lncRNAs, the coding genes located within 100 $\mathrm{kb}$ upstream/downstream of lncRNAs were checked. Further, 
we computed the expressed correlation coefficients between IncRNAs and their neighboring genes by Spearman method, in which, the significant lncRNA-mRNA correlation pairs with p-value $<0.05$ were assigned.

To investigate the functions of DE IncRNAs, we performed gene ontology (GO) and Kyoto encyclopedia of genes and genomes (KEGG) enrichments using KOBAS (http://kobas. cbi.pku.edu.cn/kobas3/genelist/) [23]. The GO terms and pathways with $\mathrm{p}$-value $<0.05$ were considered significant.

\section{Module construction base on WGCNA}

WGCNA is a systematic in silico method for analysis of complex gene regulatory networks, which can be used for finding modules of significantly correlated genes, and for relating modules to one another and to external sample traits $[24,25]$. Here, we conducted WGCNA to detect significant modules by WGCNA R package. In significant modules, we screened the lncRNAs and genes, which might be candidates to impact the swine lactation developing, milk fat and protein metabolism, and colostrum function.

\section{RESULTS}

\section{Identification of IncRNAs}

In this study, RNA-seq data of 15 samples from mammary gland of three crossbred sows with five time points were used (Supplementary Table S1) [7]. After quality control, reads mapping and lncRNA identification, a total of $1,346 \operatorname{lncRNA}$ transcripts located in 1,084 lncRNA loci were detected (Supplementary Table S2), in which, 483 (613 lncRNA transcripts) were novel lncRNAs, and 601 (733 lncRNA transcripts) were known lncRNAs. Additionally, we used the expression of lncRNAs to perform principal component analysis (PCA) analysis, and the result is shown in Figure 1B-C, in which, PC1 and PC2 could separate the samples by phenotype of stages of lactation, and PC2 and PC3 could separate the samples by individuals.

\section{Differential expression analysis}

A total of $286 \operatorname{lncRNAs}$ (Supplementary Table S3) were DE (adjusted p-value $<0.05$ ), including 9 ( 7 up-regulated and 2 down-regulated), 71 (41 up-regulated and 30 down-regulated), 169 (101 up-regulated and 68 down-regulated) and 206 (128 up-regulated and 78 down-regulated) for -10 vs -14 , -6 vs $-14,-2$ vs -14 , and 1 vs -14 comparison groups, respectively (Figure 2A-D). Notably, six lncRNAs were DE in all four comparison groups (Figure 3). Further, we performed the gap statistics analysis for 286 DE IncRNAs, and found that $\mathrm{k}=14$ was the optimal choice for cluster analysis. The result of gap statistics analysis is shown in Supplementary Figure S1. We examined the expression pattern of DE IncRNAs across five lactation stage using $\mathrm{k}$-means clustering, and these
lncRNAs could be divided into 14 distinct clusters. In cluster $4,7,9,10$, and 12, most of DE lncRNAs expressions were rapidly decreased at day -6 and day -2 , and strongly increased at day +1 , which indicated these lncRNAs were dynamic and changed from colostrogenesis to lactogenesis (Figure 4).

\section{Enrichment analysis of GWAS signals}

To assess whether mammary lncNRAs identified in this study were associated with GWAS signals, we performed enrichment analysis of sow milk ability trait for 1,084 detected lncRNAs and 286 DE lncRNAs, separately. For comparison, 10,000 random SNPs sets were generated for the tested lncRNAs. Compared to the 10,000 randomly selected SNP sets, the enrichments of 1,084 lncRNAs including 1,571 SNPs were higher for milk ability trait with enrichment fold 3.9490 ( $\mathrm{p}=$ 0.0524), and the enrichments of $286 \mathrm{DE}$ lncRNAs containing 485 SNPs were significantly enriched with GWAS signals of milk ability ( $p=0.0116)$, and the enrichment fold was 5.0546 .

\section{Prediction of target genes}

To investigate probable roles of the lncRNAs in swine mammary gland, we predicted the target genes of lncRNAs. First, a search for protein-coding genes within $100 \mathrm{~kb}$ upstream/ downstream of lncRNAs, and 6,589 genes were identified. Further, the expressions correlation between lncRNAs and protein-coding genes were examined, and 804 significant lncRNA-mRNA correlation pairs $(\mathrm{p}<0.05)$ were detected, including 685 protein coding genes and $443 \mathrm{lncRNAs}$. Among the 443 lncRNAs, 149 were DE, including 5, 41, 94, and 109 DE $\operatorname{lncRNAs}$ respectively corresponded to -10 vs $-14,-6$ vs $-14,-2$ vs -14 , and +1 vs -14 (Supplementary Table S4).

In addition, we found that 256 protein-coding genes within $100 \mathrm{~kb}$ upstream/downstream of the $149 \mathrm{DE}$ lncRNAs were also confirmed to be strongly correlated with them. To assess the function of these 286 DE lncRNAs, GO and KEGG enrichment analyses of the target genes were performed with KOBAS. There were 311 significant enrichments were presented ( $\mathrm{p}<0.05$; Supplementary Table S5), including 298 GO terms and 13 KEGG pathways. These GO and KEGG enrichments were involved in 87 genes, and most of them were directly related to the labor and colostrum. For example, CSN3, $\mathrm{SMO}$, and CSN2 were involved in mammary associated activities. In addition, we performed functional annotation of the lncRNAs in cluster 4, 7, 9, 10 and 12, and found that 107 significant enrichments $(\mathrm{p}<0.05$; Supplementary Table S6) were mainly involved in the process from colostrogenesis to lactogenesis, such as tight junction, response to progesterone, lactation, and ATP binding, which impact.

\section{Module construction}

To explore the specific lncRNAs or genes highly associated 

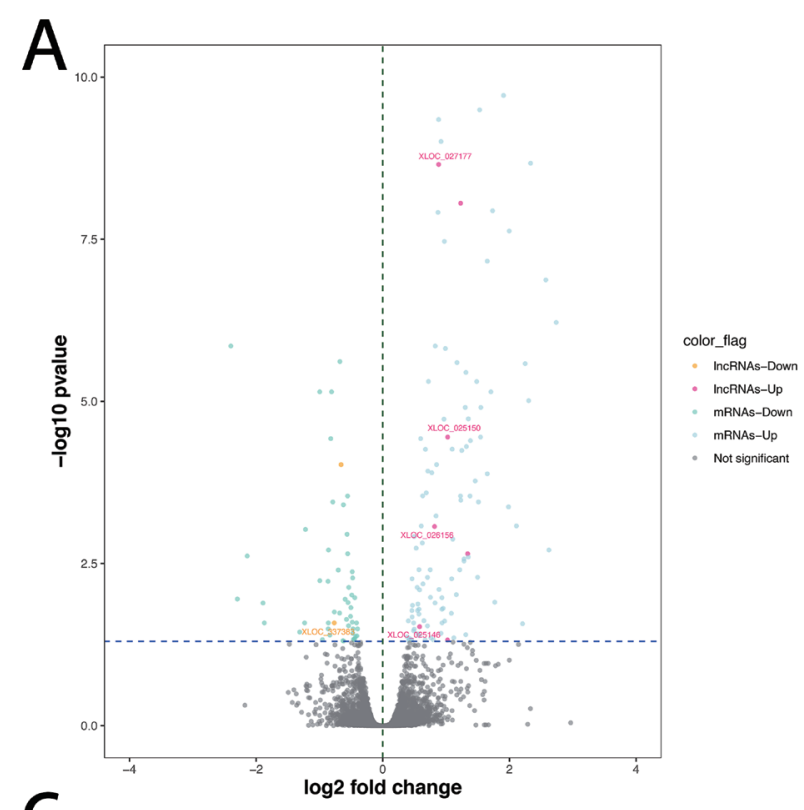

B
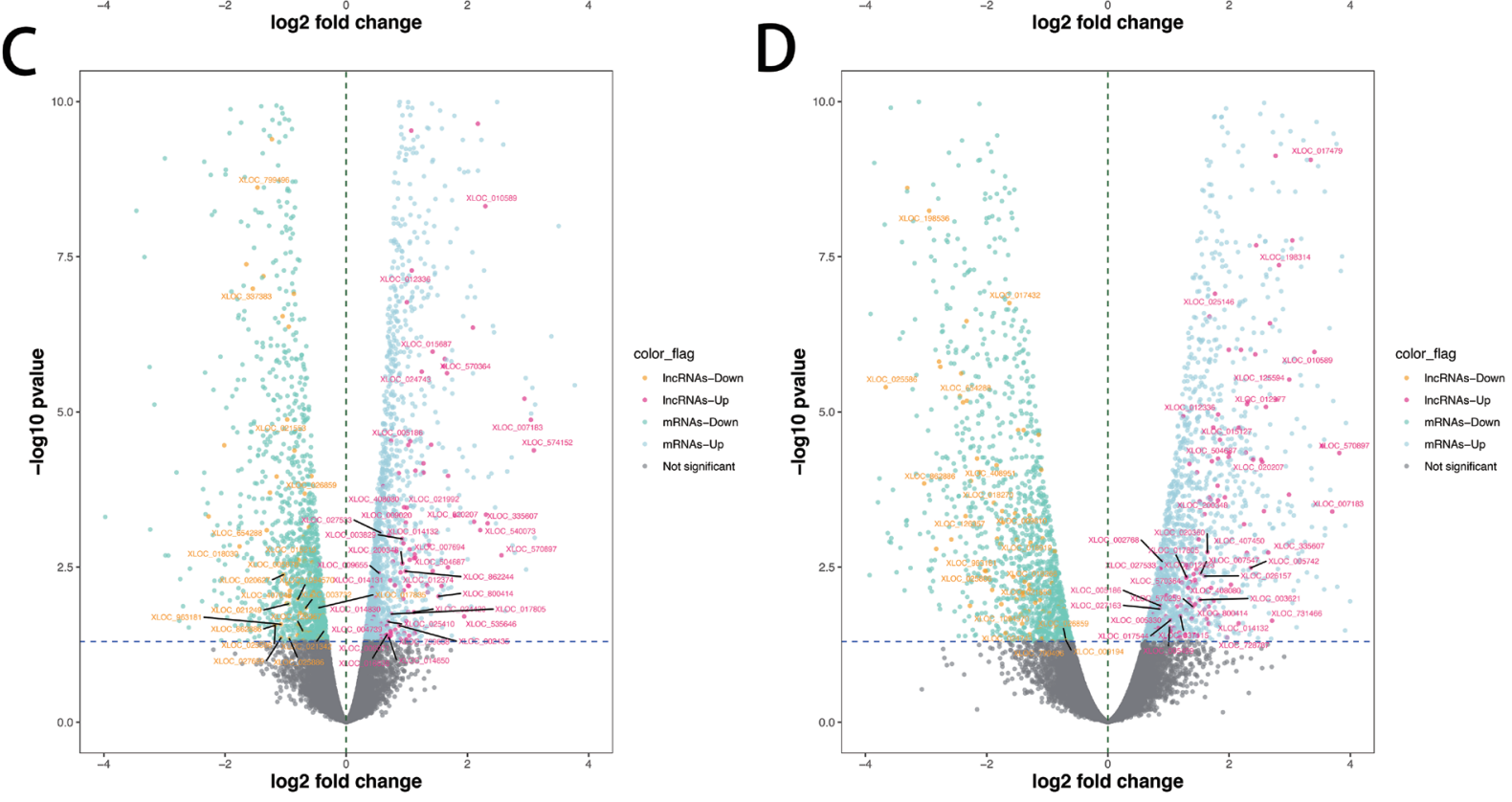

Figure 2. Identification of DE IncRNAs and mRNAs. (A) Volcano plot dispalys DE IncRNAs and mRNAs of day -10 vs day -14. (B) Volcano plot dispalys DE IncRNAs and mRNAs of day -6 vs day -14. (C) Volcano plot dispalys DE IncRNAs and mRNAs of day -2 vs day -14 . (D) Volcano plot dispalys DE IncRNAs and mRNAs of day +1 vs day -14 . The novel DE IncRNAs was marked. DE, differentially expressed.

with lactation, we performed WGCNA using DE lncRNAs and their target genes. A total of 12 modules associated with the specific expression profiles of different samples were identified (Figure 5A). Then, we calculated associations of each module with five lactation stages, and found seven modules including Greenyellow, Green, Black, Yellow, Brown, Blue, and Turquoise, were significantly associated with day -14 , day -2 , and day $+1(\mathrm{p}<0.05$; Figure $5 \mathrm{~B})$. In detail, the Greenyellow module, including 12 lncRNAs and 6 target genes were highly associated with day $-2(\mathrm{p}=0.02)$. The Green (including 5 lncRNAs and 30 target genes), Black (including 1
lncRNAs and 23 target genes), Yellow (including $5 \operatorname{lncRNAs}$ and 49 target genes), and Brown (including 1 lncRNAs and 53 target genes) modules were significantly associated with the mammary gland samples at day +1 ( $\mathrm{p}=3 \mathrm{E}-04$ to 0.009$)$. The Turquoise (including 9 lncRNAs and 91 target genes) and Blue (including 2 lncRNAs and 67 target genes) modules were strongly associated with at day -14 and day +1 ( $\mathrm{p}$ $=0.004$ to 0.04 ). The lncRNAs and target genes involved in the significant modules are shown in Supplementary Table S7. 


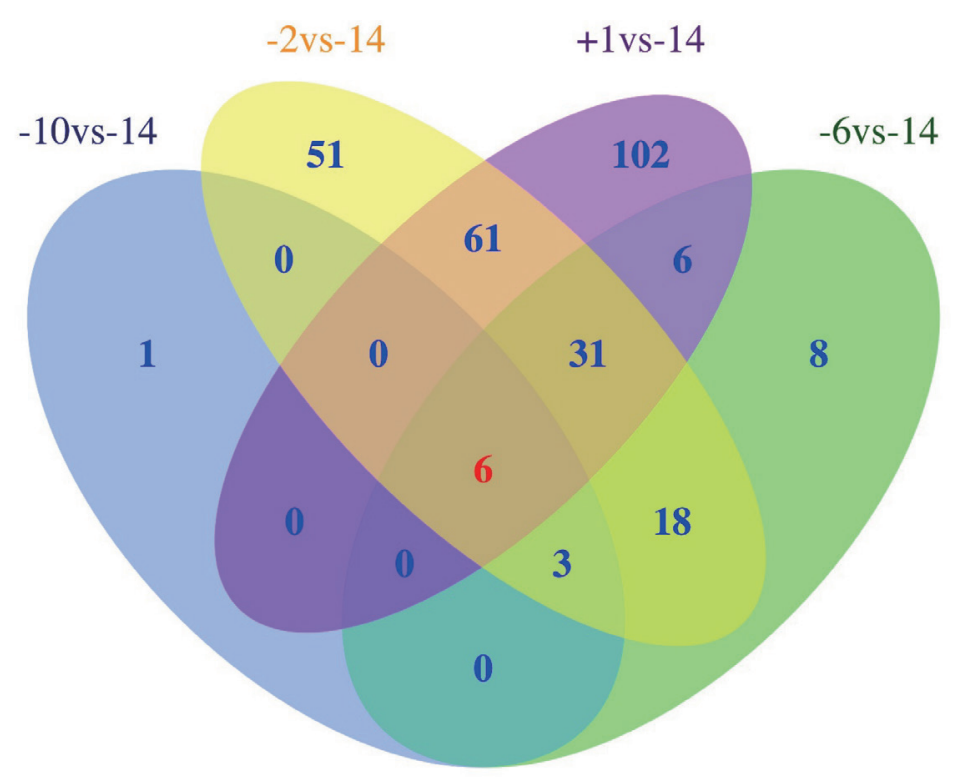

Figure 3. Venn diagram shows the number of differentially expressed (DE) IncRNAs overlapping in different swine mammary gland development stages or unique to each developmental stage. -14 , at days 14 before parturition; -10 , at days 10 before parturition; -6 , at days 6 before parturition; -2 , at days 2 before parturition; +1 , at days 1 after parturition.

\section{Comprehensive analysis}

Among 256 target genes, 121 were DE across five lactation stages, and function annotation revealed that 25 of them targeted by 23 lncRNAs were involved in lactation developing, milk fat metabolism and colostrum function (Table 1). Interestingly, 20 target genes (ACTN4, ADCY1, CSN3, SMO, PTK7, MPDZ, NPR1, CSN2, ATP2C2, PRKAG2, CD36, NPR1, ACSL3, GALNT15, C6H1orf210, CDC20, SNRPD1, FIBCD1, $A C T B$, and GALNT7) and 3 lncRNAs (XLOC_025150, ENSSS CG00000011196, and XLOC_010589) also belong to significant modules by WGCNA. These 20 target genes corresponded to 18 lncRNAs, which also included the 3 lncRNAs (XLOC_ 025150, ENSSSCG00000011196, and XLOC_010589) involved in significant modules. Hence, we suggested that these 18 lncRNAs targeting 20 genes were the candidates involved in lactation of sows, which were considered as the potentially functional candidates impacting mammary gland development from colostrogenesis to lactogenesis. In addition, we conducted the network of promising candidate lncRNAs, genes and pathways (Figure 6), in which, the functions of lncRNAs were clearly shown. For example, XLOC_025150 might regulate CSN3 gene involved in the mammary gland development and lactation.

\section{DISCUSSION}

In this study, we systematically analyzed the RNA-seq data of 15 swine mammary gland samples collected at days 14 , 10,6 , and 2 before (-) parturition to day 1 after $(+)$ parturition and identified a total of 286 DE lncRNAs targeting 256 genes. Further, we found that these DE lncRNAs had significant association with sow lactation, and were involved in delivery and lactation development, milk lipid metabolism, and immune function of colostrum, and $18 \mathrm{lncRNAs}$ targeting 20 genes which were proposed to be the candidates involved in lactation of sow.

At parturition, the preparation for copious milk synthesis and secretion have begun $[7,26]$, and the mammary gland can reach the greatest degree of structural development. In this study, we performed the validation of GWAS signals, and showed that the DE lncRNAs were significantly enriched with GWAS signals of sow milk ability, which indicated that these dynamically changed lncRNAs in different periods of mammary gland progression may be involved in milk ability. We examined the expression pattern of DE lncRNAs, most of the lncRNAs expressions were strongly changed from day -6 to day +1 . Further, 18 promising functional $\operatorname{lncRNAs}$ targeting 20 genes were mainly identified in -2 vs -14 and 1 vs -14 groups, which reflected a strong activation of many metabolic processes before and after parturition. In the WGCNA, 20 promising functional DE target genes were involved in the significant modules, which were highly associated with the mammary gland samples at day -14 , day -2 , and day +1 . Hence, our results were consistent with the concept: before and after farrowing with the formation of colostrum, mammary glands immediately undergo strong functional differentiation [5].

In general, lncRNAs exert regulatory functions at different levels of gene expression to influence the tumor growth, cell-cycle, and apoptosis [24,27]. For example, lncRNA H19 

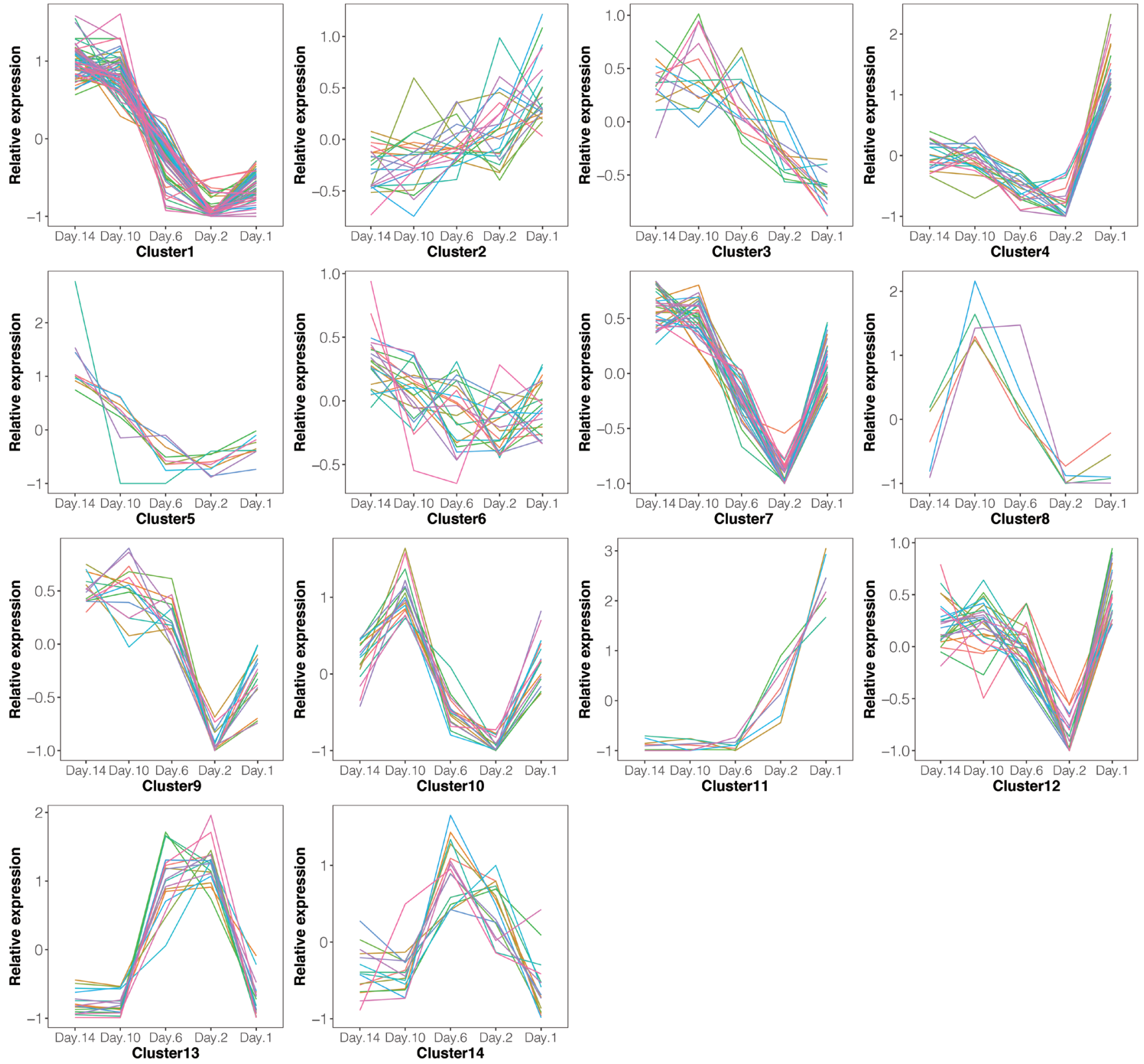

Figure 4. Expression pattern of differentially expressed (DE) IncRNAs across five stages. The colored lines represent that the different IncRNA changes.

is involved in regulation of high glucose-induced apoptosis through targeting VDAC1 [28]. Here, 11 lncRNA-mRNA target pairs, XLOC_020627-ACTN4, ENSSSCG00000051193ADCY1,XLOC_025150-CSN3, ENSSSCG00000042618-SMO, XLOC_963181-PTK7, ENSSSCG00000051701-MPDZ, XLOC_018030-NPR1, XLOC_025146-CSN2, ENSSSCG00 000041015-ATP2C2, ENSSSCG00000046607-PRKAG2, and ENSSSCG00000049698-ACTB, were involved in delivery and lactation metabolism, such as tight junctions, oxytocin, development of the mammary gland and lactation. Tight junctions of mammary gland from the pregnant animal are leaky, undergoing closure around parturition to become the impermeable tight junctions of the lactating animal [29]. In dairy cattle, after parturition, the start of copious milk production requires the closure of tight junctions to form the blood-milk barrier and prevent paracellular transfer of blood constituents into milk (such as lactate dehydrogenase and serum albumin) and vice versa (such as appearance of $\alpha$-lactalbumin in blood) [30]. Oxytocin is a nonapeptide hormone that has a central role in the regulation of parturition and lactation [31], and its best-known and most well-established roles are stimulation of uterine contractions during parturi- 
A

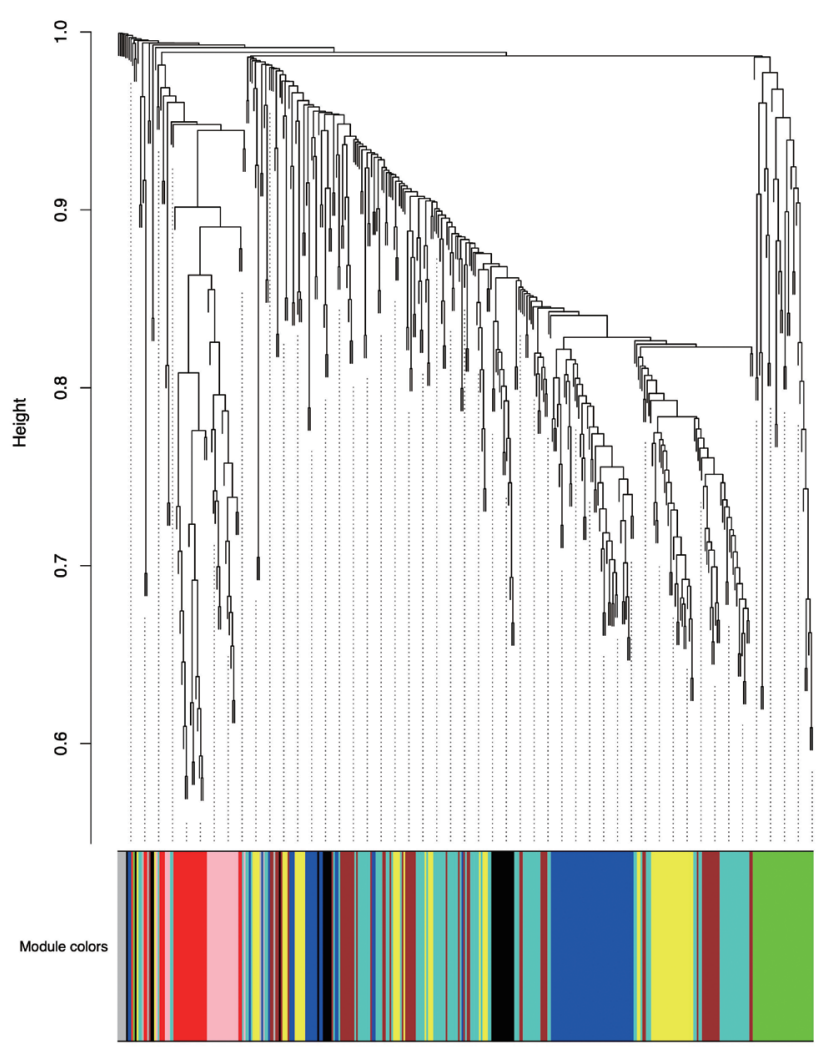

B

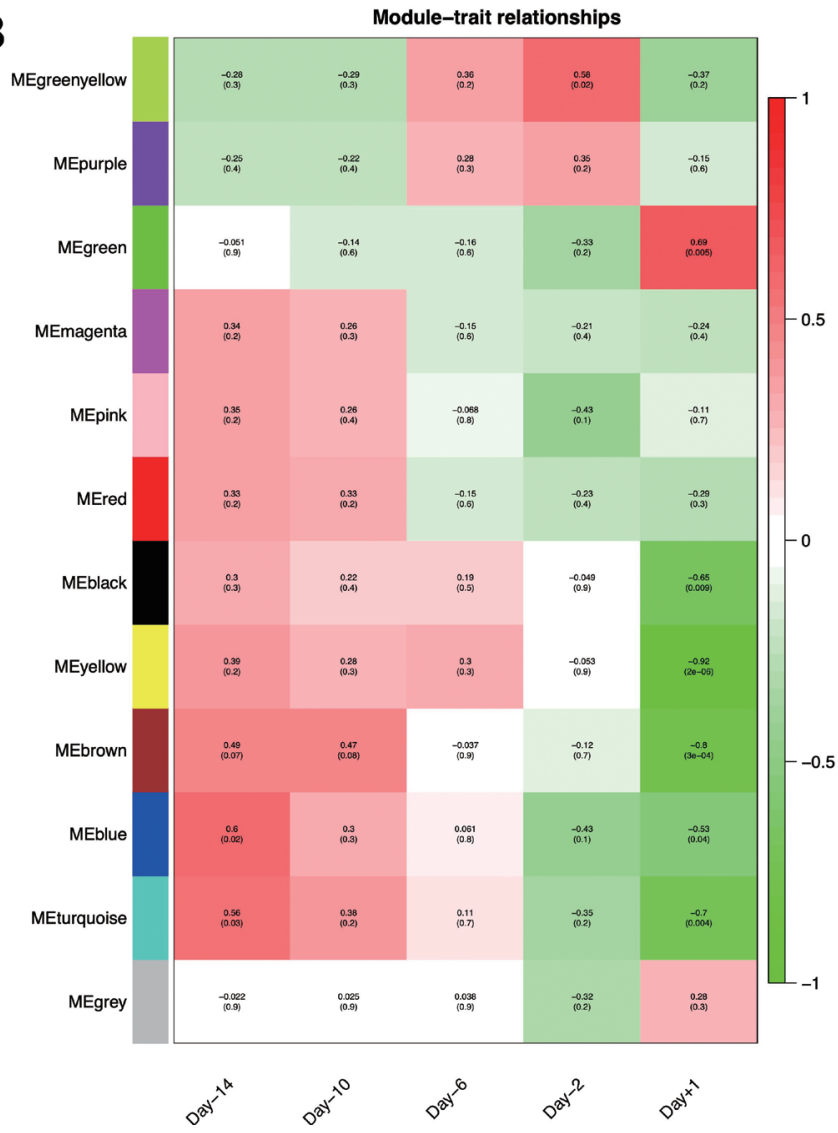

Figure 5. WGCNA at each swine mammary gland development. (A) Hierarchical cluster tree showing co-expression modules identified by WGCNA. (B) Module-sample relationships. Each column indicates a sample, and each row represents a module. The correlation coefficient and p-value of each sample-module relationship are displayed. Red represents high correlation value, and green represents low correlation value. WGCNA, weighted gene co-expression network analysis.

tion and milk release during lactation [32]. Development of the mammary gland occurs in defined stages, which are connected to sexual development and reproduction (embryonic, prepubertal, pubertal, pregnancy, lactation, and involution) [33]. Lactation is a highly demanding lipid synthesis and transport process that is crucial for the development of newborn mammals [34]. Embryo development ending in birth or egg hatching term was defined as the process whose specific outcome is the progression of an embryo over time, from zygote formation until the end of the embryonic life stage, and for mammals it is usually considered to be birth. The above reports suggested that these GO and KEGG enrichments were related with the parturition and lactation. Hence, we proposed that these lncRNAs in 11 lncRNA-mRNA target pairs might be regulated their target genes to impact the delivery and lactation processes.

Additionally, we found eight lncRNA-mRNA target pairs, XLOC_020627-ACTN4, ENSSSCG00000051193-ADCY1, ENSSSCG00000042618-SMO, XLOC_963181-PTK7, XLOC_ 026156-CD36, XLOC_018030-NPR1, XLOC_010589-ACSL3, and ENSSSCG00000046607-PRKAG2, were enriched in per- oxisome proliferator-activated receptors (PPARs), regulation of lipolysis in adipocytes, cellular response to lipid, and adipocytokine signaling pathways, that might be related with the milk components metabolism.

For the piglets, colostrum plays an important role in ensuring their survival, growth and health by providing energy, nutrients, immunoglobulins, growth factors and many other bioactive components and cells [35]. In the present study, we found that 12 lncRNA-mRNA target pairs, XLOC_020627ACTN4, ENSSSCG00000042618-SMO, XLOC_963181-PTK7, ENSSSCG00000011196-GALNT15, ENSSSCG00000041987C6H1orf210, ENSSSCG00000041987-CDC20, ENSSSCG 00000041015-ATP2C2, ENSSSCG00000046607-GALNTL5, ENSSSCG00000042000-SNRPD1, XLOC_002435-FIBCD1, ENSSSCG00000049698-ACTB, and ENSSSCG000000482 64-GALNT7, were involved in leukocyte transendothelial migration, response to retinoic acid, drug binding, mucin type O-glycan biosynthesis, regulation of defense response to virus, and positive regulation of ubiquitin-protein transferase activity enrichments, which might impact the immune function of colostrum. 
Table 1. GO and KEGG enrichments of 23 potentially functional IncRNAs and their 25 target genes

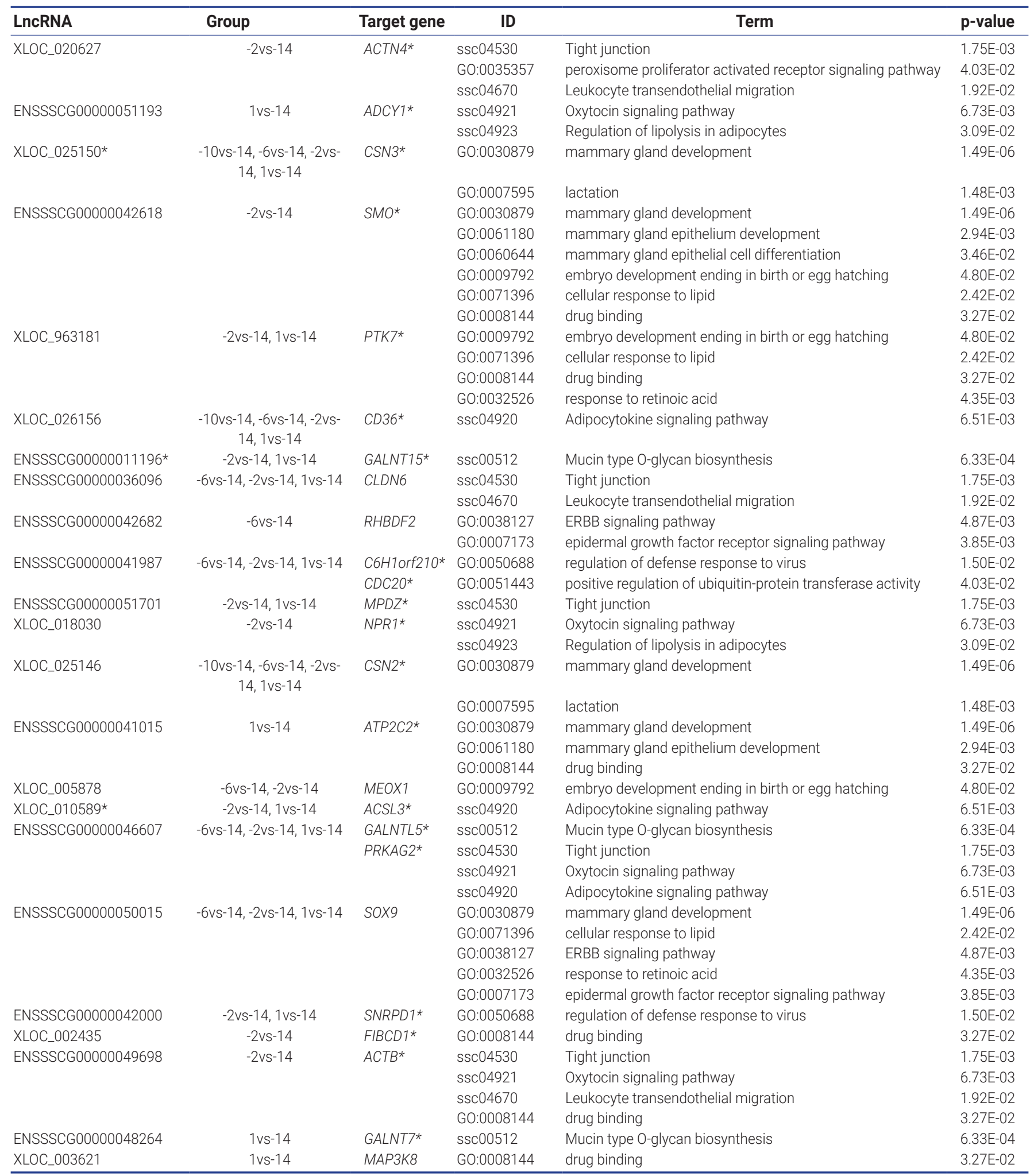

GO, gene ontology; KEGG, Kyoto encyclopedia of genes and genomes.

* Indicates the IncRNAs and their target genes involved in the significant module by WGCNA.

Based on these advantages, we proposed these $18 \operatorname{lncRNAs}$ (XLOC_020627, ENSSSCG00000051193, XLOC_025150, ENSSSCG00000042618, XLOC_963181, ENSSSCG000000
51701,XLOC_018030, XLOC_025146, ENSSSCG00000041015, ENSSSCG00000046607, ENSSSCG00000049698, XLOC_ 026156, XLOC_010589, ENSSSCG00000011196, ENSSSCG 

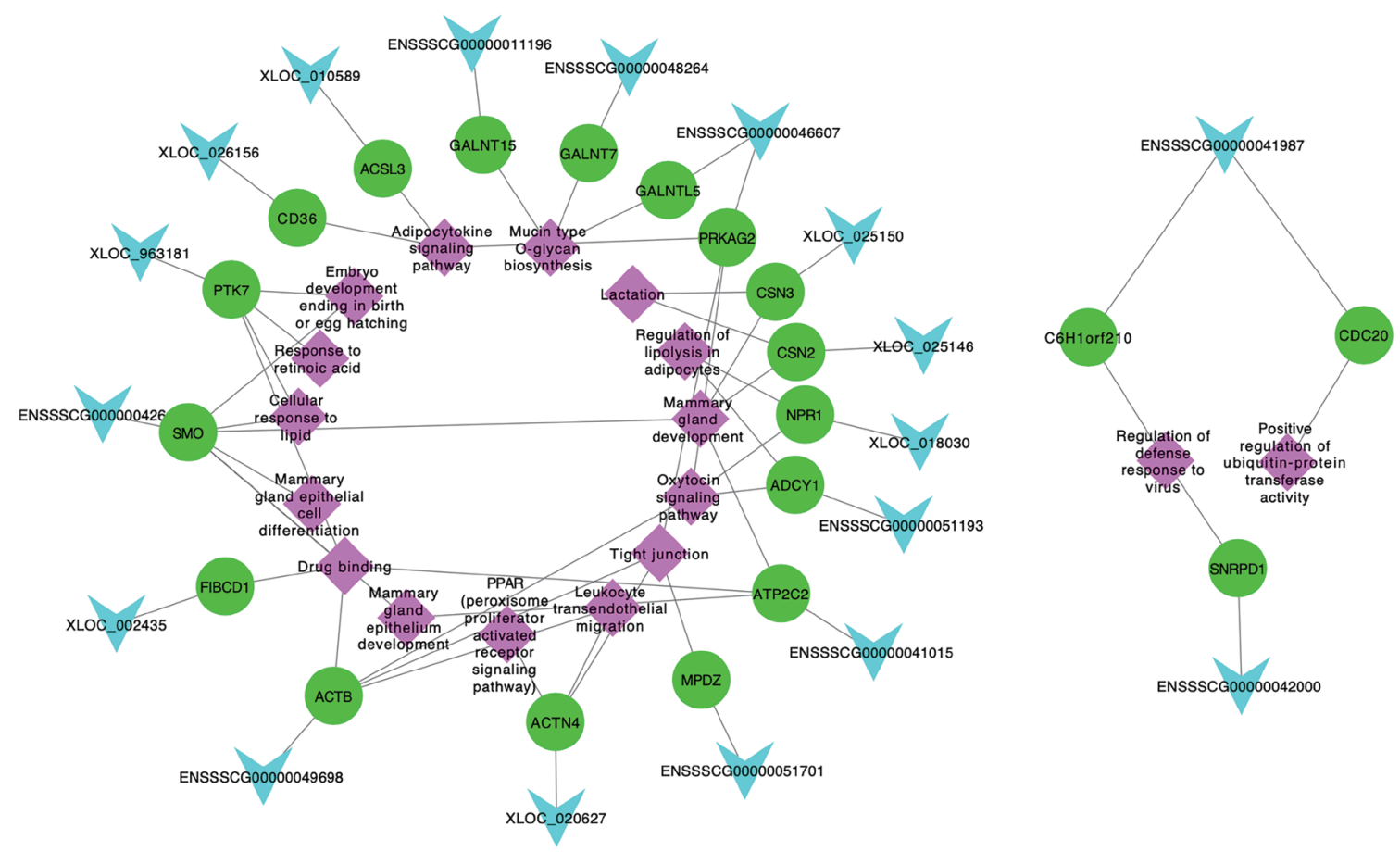

Figure 6. Network plot of the promising candidate IncRNAs, genes and pathways. The light blue, green and purple indicate IncRNAs, genes and pathways, respectively.

00000041987, ENSSSCG00000042000, XLOC_002435, and ENSSSCG00000048264) as the promising candidates for swine parturition and lactation. Our network results of 18 lncRNAs, their 20 target genes and corresponding pathways further clearly showed the functions of the candidate lcnRNAs and their target genes. There is a high probability that these lncRNAs and genes interact closely and thus act as key regulators in mammary gland, thus, future work should focus on the potential roles of them in sow lactation.

Sow milk production is the major factor which can limit growth and survival of piglets. To enhance milk performance, it is necessary to understand the process of mammary gland morphogenesis and to identify the regulatory factors of mammary development. In this study, we identified 1,084 lncRNAs in swine mammary gland using RNA-seq data, and 286 were DE lncRNAs in five lactation stages. Further, the enrichment analysis of GWAS signals for sow milk ability trait validated that the identified DE IncRNAs had significant association with milk ability trait. Integrated analysis of the DE IncRNAs expression pattern examination, targets prediction, function annotation and WGCNA, we proposed that $18 \mathrm{lncRNAs}$ (such as XLOC_020627, ENSSSCG00000051193, XLOC_025150, ENSSSCG00000042618, XLOC_963181, ENSSSCG000000 51701, XLOC_018030, and XLOC_025146) targeting 20 genes (such as ACTN4, ADCY1, CSN3, SMO, CSN2, PRKAG2, $F I B C D 1$, and GALNT7) as promising candidates which were involved in swine parturition and colostrum occurrence processes. These results provided a new insight for exploring critically regulatory factors involved in reproductive performance of sow.

\section{CONFLICT OF INTEREST}

We certify that there is no conflict of interest with any financial organization regarding the material discussed in the manuscript.

\section{FUNDING}

This research was supported by the China Agriculture Research System of MOF and MARA, and Chinese Academy of Agricultural Sciences Foundation (20118/2020-YWFYTS-8).

\section{ACKNOWLEDGMENTS}

We acknowledge financial support by the Chinese Academy of Agricultural Sciences.

\section{SUPPLEMENTARY MATERIAL}

Supplementary file is available from: https://doi.org/10.5713/ ab. 21.0308 
Supplementary Figure $\mathbf{S 1}$. The result of gap statistics analysis. $\mathrm{K}=$ 14 was the optimal choice for cluster analysis.

Supplementary Table S1. The information of RNA sequencing data for 15 samples of sow mammary gland tissue

Supplementary Table S2. The results of the identified IncRNAs in this study

Supplementary Table S3. The detailed information of DE IncRNAs in this study

Supplementary Table S4. The predicted target genes of DE IncRNAs Supplementary Table S5. Significant GO and KEGG pathways of the target genes of DE IncRNAs

Supplementary Table S6. Significant GO and KEGG pathways of IncRNAs in cluster 4, 7, 9, 10 and 12

Supplementary Table S7. The IncRNAs and genes involved in significant modules

\section{REFERENCES}

1. Kim S. Mammary gland growth and nutrient mobilization in lactating sows: a dynamic model to describe nutrient flow. Champaign, IL, USA: University of Illinois; 1999.

2. Boyd RD, Kensinger RS. Metabolic precursors for milk synthesis. Wageningen, The Netherlands: Wageningen Press; 1998.

3. Quesnel H, Farmer C, Devillers N. Colostrum intake: Influence on piglet performance and factors of variation. Livest Sci 2012;146:105-14. https://doi.org/10.1016/j.livsci.2012. 03.010

4. Vadmand CN, Krogh U, Hansen CF, Theil PK. Impact of sow and litter characteristics on colostrum yield, time for onset of lactation, and milk yield of sows. J Anim Sci 2015; 93:2488-500. https://doi.org/10.2527/jas.2014-8659

5. Hurley WL. Review: Mammary gland development in swine: embryo to early lactation. Animal 2019;13:S11-9. https://doi. org/10.1017/S1751731119000521

6. Balzani A, Cordell HJ, Sutcliffe E, Edwards SA. Heritability of udder morphology and colostrum quality traits in swine. J Anim Sci 2016;94:3636-44. https://doi.org/10.2527/jas.20160458

7. Palombo V, Loor JJ, D'Andrea M, et al. Transcriptional profiling of swine mammary gland during the transition from colostrogenesis to lactogenesis using RNA sequencing. BMC Genomics 2018;19:322. https://doi.org/10.1186/s12864018-4719-5

8. Jin Y, Zhang K, Huang W, et al. Identification of functional lncRNAs in pseudorabies virus type II infected cells. Vet Microbiol 2020;242:108564. https://doi.org/10.1016/j.vetmic. 2019.108564

9. Liang G, Yang Y, Li H, et al. LncRNAnet: a comprehensive Sus scrofa lncRNA database. Anim Genet 2018;49:632-5. https://doi.org/10.1111/age. 12720

10.St Laurent G, Wahlestedt C, Kapranov P. The Landscape of long noncoding RNA classification. Trends Genet 2015;31: 239-51. https://doi.org/10.1016/j.tig.2015.03.007

11. Miao Z, Wang S, Zhang J, et al. Identification and comparison of long non-conding RNA in Jinhua and Landrace pigs. Biochem Biophys Res Commun 2018;506:765-71. https:// doi.org/10.1016/j.bbrc.2018.06.028

12.Huang W, Zhang X, Li A, Xie L, Miao X. Genome-wide analysis of mRNAs and lncRNAs of intramuscular fat related to lipid metabolism in two pig breeds. Cell Physiol Biochem 2018;50:2406-22. https://doi.org/10.1159/000495101

13. Wang J, Ren QL, Hua LS, et al. Comprehensive analysis of differentially expressed mRNA, lncRNA and circRNA and their ceRNA networks in the longissimus dorsi muscle of two different pig breeds. Int J Mol Sci 2019;20:1107. https:// doi.org/10.3390/ijms20051107

14.Li S, Chen C, Chai M, et al. Identification and analysis of lncRNAs by whole-transcriptome sequencing in porcine preadipocytes induced by BMP2. Cytogenet Genome Res 2019;158:133-44. https://doi.org/10.1159/000501182

15. Wang Y, Hu T, Wu L, Liu X, Xue S, Lei M. Identification of non-coding and coding RNAs in porcine endometrium. Genomics 2017;109:43-50. https://doi.org/10.1016/j.ygeno. 2016.11.007

16. Bolger AM, Lohse M, Usadel B. Trimmomatic: a flexible trimmer for Illumina sequence data. Bioinformatics 2014; 30:2114-20. https://doi.org/10.1093/bioinformatics/btu170

17.Pertea M, Pertea GM, Antonescu CM, Chang TC, Mendell JT, Salzberg SL. StringTie enables improved reconstruction of a transcriptome from RNA-seq reads. Nat Biotechnol 2015;33:290-5. https://doi.org/10.1038/nbt.3122

18. Guttman M, Garber M, Levin JZ, et al. Correction: Corrigendum: Ab initio reconstruction of cell type-specific transcriptomes in mouse reveals the conserved multi-exonic structure of lincRNAs (2010;28:503-10). Nat Biotechnol 2010;28:756. https://doi.org/10.1038/nbt0710-756b

19. Ahmad A, Dey L. A k-mean clustering algorithm for mixed numeric and categorical data. Data Knowl Eng 2007;63:50327. https://doi.org/10.1016/j.datak.2007.03.016

20.Cai WT, Li C, Li JY, Song JZ, Zhang SL. Integrated small RNA sequencing, transcriptome and GWAS data reveal microRNA regulation in response to milk protein traits in Chinese Holstein cattle. Front Genet 2021;12:726706.

21.Guil S, Esteller M. Cis-acting noncoding RNAs: friends and foes. Nat Struct Mol Biol 2012;19:1068-75. https://doi.org/ 10.1038/nsmb. 2428

22.Derrien T, Johnson R, Bussotti G, et al. The GENCODE v7 catalog of human long noncoding RNAs: analysis of their gene structure, evolution, and expression. Genome Res 2012;22:1775-89. https://doi.org/10.1101/gr.132159.111

23.Xie C, Mao X, Huang J, et al. KOBAS 2.0: a web server for annotation and identification of enriched pathways and diseases. Nucleic Acids Res 2011;39(Suppl_2):W316-22. https://doi.org/10.1093/nar/gkr483

24.Le K, Guo H, Zhang Q, et al. Gene and lncRNA co-expression network analysis reveals novel ceRNA network for triple- 
negative breast cancer. Sci Rep 2019;9:15122. https:/doi.org/ 10.1038/s41598-019-51626-7

25.Langfelder P, Horvath S. WGCNA: an R package for weighted correlation network analysis. BMC Bioinformatics 2008;9: 559. https://doi.org/10.1186/1471-2105-9-559

26. Kensinger RS, Collier RJ, Bazer FW, Ducsay CA, Becker HN. Nucleic acid, metabolic and histological changes in gilt mammary tissue during pregnancy and lactogenesis. J Anim Sci 1982;54:1297-308. https://doi.org/10.2527/jas1982.5461 297x

27.Wang L, Cho KB, Li Y, Tao G, Xie Z, Guo B. Long noncoding RNA (lncRNA)-mediated competing endogenous RNA networks provide novel potential biomarkers and therapeutic targets for colorectal cancer. Int J Mol Sci 2019;20: 5758. https://doi.org/10.3390/ijms20225758

28.Li X, Wang H, Yao B, Xu W, Chen J, Zhou X. IncRNA H19/ miR-675 axis regulates cardiomyocyte apoptosis by targeting VDAC1 in diabetic cardiomyopathy. Sci Rep 2016;6:36340. https://doi.org/10.1038/srep36340

29. Nguyen DA, Neville MC. Tight junction regulation in the mammary gland. J Mammary Gland Biol Neoplasia 1998;3: 233-46. https://doi.org/10.1023/a:1018707309361
30.Kessler EC, Wall SK, Hernandez LL, Gross JJ, Bruckmaier RM. Short communication: Mammary gland tight junction permeability after parturition is greater in dairy cows with elevated circulating serotonin concentrations. J Dairy Sci 2019;102:1768-74. https://doi.org/10.3168/jds.2018-15543

31.Arrowsmith S, Wray S. Oxytocin: its mechanism of action and receptor signalling in the myometrium. J Neuroendocrinol 2014;26:356-69. https://doi.org/10.1111/jne. 12154

32. Gimpl G, Fahrenholz F. The oxytocin receptor system: structure, function, and regulation. Physiol Rev 2001;81:629-83. https://doi.org/10.1152/physrev.2001.81.2.629

33. Hennighausen L, Robinson GW. Signaling pathways in mammary gland development. Dev Cell 2001;1:467-75. https://doi.org/10.1016/s1534-5807(01)00064-8

34. Wan YH, Saghatelian A, Chong LW, Zhang CL, Cravatt BF, Evans RM. Maternal PPAR gamma protects nursing neonates by suppressing the production of inflammatory milk. Gene Dev 2007;21:1895-908. https://doi.org/10.1101/gad.1567207

35. Quesnel H, Farmer C. Review: nutritional and endocrine control of colostrogenesis in swine. Animal 2019;13:S26-34. https://doi.org/10.1017/S1751731118003555 